\title{
The Ability of Heart Failure Specialists to Accurately Predict NT-proBNP Levels Based on Clinical Assessment and a Previous NT-proBNP Measurement
}

\author{
Tara L Sedlak ${ }^{1}$, Mann Chandavimol ${ }^{2}$, Anna Calleja ${ }^{3}$, Catherine Clark ${ }^{4}$, Margaret Edmonds ${ }^{5}$, \\ Aihua $\mathrm{Pu}^{6}$, Karin $\mathrm{H}$ Humphries ${ }^{7}$ and Andrew Ignaszewski*, 8 \\ ${ }^{1}$ University of British Columbia; ${ }^{2}$ University of British Columbia $;{ }^{3}$ University of British Columbia; ${ }^{4}$ St. Paul's Hospital, \\ Vancouver; ${ }^{5}$ St. Paul's Hospital, Vancouver; ${ }^{6}$ Centre for Health Evaluation \& Outcome Sciences; ${ }^{7}$ University of British \\ Columbia, Centre for Health Evaluation \& Outcome Sciences and ${ }^{8}$ University of British Columbia
}

\begin{abstract}
Background: The value of routine aminoterminal pro type B natriuretic peptide (NT-proBNP) measurements in outpatient clinics remains unknown.

Objectives: We sought to determine the accuracy with which heart failure (HF) specialists can predict NT-proBNP levels in HF outpatients based on clinical assessment.

Methods: We prospectively studied 160 consecutive HF patients followed in an outpatient multidisciplinary HF clinic. During a regular office visit, HF specialists were asked to estimate a patient's current NT-proBNP level based upon their clinical assessment and all available information from their chart, including a previous NT-proBNP level (if available). NT-proBNP estimations were grouped into prognostic categories $(<125,125-1000,1000-4998$, or $\geq 4999 \mathrm{pg} / \mathrm{mL}) \mathrm{and}$ comparisons made between actual and estimate values.

Results: Overall, HF specialists estimated $67.5 \%$ of NT-proBNP levels correctly. After adjusting for clinical characteristics, knowledge of a prior NT-proBNP measurement was the only significant predictor of estimation accuracy $(\mathrm{p}=0.01)$. Compared to patients with a prior NT-proBNP level $<125 \mathrm{pg} / \mathrm{mL}$, physicians were $95 \%$ less likely to get a correct estimation in patients with the highest prior NT-proBNP level $(\geq 4999 \mathrm{pg} / \mathrm{mL})$.

Conclusion: HF specialists are reasonably accurate at estimating current NT-proBNP levels based upon clinical assessment and a previous NT-proBNP level, if those levels were $<4999 \mathrm{pg} / \mathrm{mL}$. Likely, initial but not routine NT-proBNP measurements are useful in outpatient HF clinics.
\end{abstract}

\section{INTRODUCTION}

B-type natriuretic peptide (BNP) is released from ventricular myocardial tissue in response to increased wall stress. Both BNP and aminoterminal pro-type B natriuretic peptide (NT-proBNP), an inactive amino acid product of BNP prohormone cleavage, can be measured in the serum and both are typically elevated in heart failure (HF).

NT-proBNP is frequently measured at regular intervals in HF outpatients. Multiple studies have demonstrated the value of NT-proBNP in the diagnosis of congestive HF [1-6]. Recent research suggests that serial NT-proBNP measurements may also have a role in prognostic evaluation [7-12] and therapy optimization [13-16]. To date, however, studies have not been conducted looking at the cost-effectiveness of regular outpatient NT-proBNP measurements. Further, in the 2007 Canadian Cardiovascular Society Consensus Conference recommendations on $\mathrm{HF}$, sequential measurements of

\footnotetext{
*Address correspondence to this author at the Health Heart Program, 1081 Burrard Street, Vancouver, BC, Canada, V6Z 1Y6; Tel: 604-806-8605; Fax: 604-806-8338; E-mail: AIgnaszewski@ providencehealth.bc.ca

Institutions where work originated: University of British Columbia; Centre for Health Evaluation \& Outcome Sciences; and St. Paul's Hospital, Vancouver.
}

BNP/NT-proBNP levels were given a class IIb recommendation [17].

Skepticism remains regarding the value of routine NTproBNP measurements in outpatient clinics. Specifically, it is unknown whether clinicians are able to accurately predict NT-proBNP levels on an outpatient basis. Our primary objective was to determine the accuracy with which HF specialists can predict NT-proBNP levels in HF patients based on clinical assessment during an office visit. Our secondary objective was to determine whether certain clinical characteristics (i.e., gender, BMI, age, eGFR, NYHA, and the absence of a previous NT-proBNP level) were predictors of accurate NT-proBNP estimations.

\section{METHODS}

\section{Patient Characteristics and Study Protocol}

We enrolled 160 consecutive patients $>18$ years with known HF attending the outpatient Heart Function, PostHeart Transplant, and Maintenance Clinics at St. Paul's Hospital in Vancouver, British Columbia, Canada. Ethics approval for the study was obtained from the University of British Columbia/Providence Health Care Research Ethics Board. NT-proBNP levels were drawn from each HF patient upon arrival to clinic. Each patient was then assessed by one 
of five $\mathrm{HF}$ specialists as a regular office visit. All HF specialists were cardiologists trained specifically in HF and all patients were seen in St. Paul's Hospital, a high volume tertiary care center. During this visit, physicians were asked to estimate the patient's current NT-proBNP level based upon their clinical assessment and all available information from the patient's chart, including a previous NT-proBNP level (if available). Actual and estimated NT-proBNP levels were then recorded by an independent physician, in addition to gender, age, previous NT-proBNP level (if available), current body mass index (BMI), most recent glomerular filtration rate (eGFR), and current New York Heart Association (NYHA) class. NT-proBNP levels were grouped into prognostic categories $(<125,125-1000,1000-4998$ or $\geq 4999$ $\mathrm{pg} / \mathrm{mL}$ ) outlined by a recent study by Rothenburger et al. [10]. If the estimated and the actual NT-proBNP prognostic categories were the same, we considered this as an accurate estimation.

\section{NT-proBNP Measurement}

NT-proBNP levels were measured by the Elecsys ${ }^{\circledR}$ proBNP immunoassay on the Roche Elecsys ${ }^{\circledR} 1010$ immunoassay analyzer.

\section{Statistical Analyses}

For univariate analysis, the Chi-square test was used to examine the association between correct physician NTproBNP estimation and the following factors: gender, BMI $<25 \mathrm{~kg} / \mathrm{m}^{2}$, age $<65$, eGFR $>60 \mathrm{~mL} / \mathrm{min} . / 1.73 \mathrm{~m}^{2}$, NYHA class I or II, the absence of a previous NT-proBNP measurement, and the category of the most recent prior NTproBNP level (if available). For multivariable analysis, a logistic regression model was used to investigate the association between the prior NT-proBNP prognostic category and correct physician NT-proBNP estimation, adjusted for gender, BMI, age, eGFR, NYHA, and the time difference between the current test and prior test.

\section{RESULTS}

Baseline characteristics of the patients are displayed in Table 1. In general, more patients were male $(69.5 \%)$, less than 65 years of age $(56.1 \%)$, had an elevated BMI $\geq 25$ (76.2\%), were NYHA I or II (76.4\%), and had a previous NT-proBNP $(82.3 \%)$. Most patients with a previous NTproBNP level had a measurement between 1000-4998 pg/mL and a time interval between measurements of 6 to 12 months.

Overall, HF specialists estimated $67.5 \%$ of NT-proBNP levels correctly. In univariate analysis, a prior NT-proBNP measurement was significantly associated with estimation accuracy $(p=0.01)$. Importantly, the level of prior NTproBNP measurement was significantly associated with estimation accuracy $(\mathrm{p}=0.002)$. For patients with a prior NTproBNP test, physician estimations were good for patients with a prior BNP level $<125 \mathrm{pg} / \mathrm{mL}(92.3 \%)$ and BNP levels between $125-999 \mathrm{pg} / \mathrm{mL}(84.2 \%)$, but the accuracy reduced greatly as the prior NT-proBNP levels increased. The correct estimation was only $66.7 \%$ in patients with a prior BNP level between 1000 and $4998 \mathrm{pg} / \mathrm{mL}$. The accuracy of physician estimations for patients with a prior NT-proBNP level $\geq 4999$ further reduced to $47.1 \%$, which was similar to that of pa- tients without a prior NT-proBNP test (46.2\%). No significant association was observed for any other factors.

Table 1. Baseline Characteristics of HF Patients

\begin{tabular}{|c|c|c|}
\hline Characteristic & $\mathbf{n}$ & \% \\
\hline \hline Female & 50 & 30.5 \\
\hline BMI $\geq 25 \mathrm{~kg} / \mathrm{m}^{2}$ & 125 & 76.2 \\
\hline Age $\geq 65$ years & 72 & 43.9 \\
\hline eGFR $\geq 60$ mL/min./1.73m ${ }^{2}$ & 85 & 51.8 \\
\hline NYHA III/IV & 38 & 23.6 \\
\hline Prior NT-proBNP measurement & 135 & 82.3 \\
\hline Time from last measurement & & \\
\hline$<6$ month & 39 & 28.9 \\
\hline 6 month to 12 month & 61 & 45.2 \\
\hline$\geq 12$ month & 35 & 25.9 \\
\hline Prior NT-proBNP level (pg/mL) & & \\
\hline$<125$ & 13 & 9.6 \\
\hline $125-1000$ & 42 & 31.1 \\
\hline $1000-4999$ & 63 & 46.7 \\
\hline$\geq 4999$ & 17 & 12.6 \\
\hline
\end{tabular}

$\mathrm{BMI}=$ body mass index , eGFR $=$ glomerular filtration rate NYHA $=$ New York Heart Association; NT-proBNP = aminoterminal pro type $\mathrm{B}$ natriuretic peptide.

The results of multivariable analysis echoed that of the univariate analysis. The absence of a prior NT-proBNP measurement significantly reduced the accuracy of physician estimation (OR: 0.05; 95\%CI 0.004-0.49) (Table 2). The level of prior NT-proBNP also greatly affected the estimation accuracy. Compared to patients with a prior NT-proBNP level $<125 \mathrm{pg} / \mathrm{mL}$, physicians were $95 \%$ less likely to get a correct estimation, in patients with the highest prior NTproBNP level $(\geq 4999 \mathrm{pg} / \mathrm{mL})$. This was after adjustment for gender, BMI, age, GFR, NYHA, and the time difference between the current and prior test.

\section{DISCUSSION}

We found that HF specialists are relatively accurate at estimating NT-proBNP prognostic categories based upon clinical assessment and a previous NT-proBNP level. They are less accurate at predicting NT-proBNP levels in new patients with no prior NT-proBNP measurement and in patients with a prior NT-proBNP level $\geq 4999 \mathrm{pg} / \mathrm{mL}$. Renal insufficiency, NYHA class, BMI, gender, and age do not appear to be predictive markers of accurate NT-proBNP estimations. To our knowledge, we are the first group to establish that perhaps initial but not routine NT-proBNP measurements may be useful in outpatients whose initial measurements were < $4999 \mathrm{pg} / \mathrm{mL}$.

\section{NT-proBNP/BNP in the Diagnosis, Prognostic Evalua- tion, and Management of Patients with CHF}

To date, NT-proBNP/BNP has been studied in a variety of clinical settings. In the diagnosis of congestive HF, the 
Table 2. Adjusted Odds Ratios of Correct Estimation of Current NT-proBNP Category

\begin{tabular}{|c|c|c|c|}
\hline No prior NT-proBNP measurement & 0.05 & $(0.004,0.49)$ & 0.01 \\
\hline$\geq 12$ months & 1.00 & & \\
\hline \multicolumn{4}{|l|}{ Prior NT-proBNP level (pg/mL) } \\
\hline$<125$ & 1.00 & & \\
\hline $125-999$ & 0.15 & $(0.016,1.377)$ & 0.09 \\
\hline BMI $\geq 25 \mathrm{~kg} / \mathrm{m}^{2}$ & 1.16 & $(0.456,2.958)$ & 0.75 \\
\hline Age $\geq 65$ & 1.03 & $(0.424,2.515)$ & 0.94 \\
\hline $\mathrm{eGFR} \geq 60 \mathrm{~mL} / \mathrm{min} . / 1.73 \mathrm{~m}^{2}$ & 0.53 & $(0.191,1.483)$ & 0.23 \\
\hline NYHA class III/IV & 0.69 & $(0.288,1.657)$ & 0.41 \\
\hline
\end{tabular}

$\mathrm{BMI}=$ body mass index $;$ eGFR = glomerular filtration rate; NYHA = New York Heart Association; NT-proBNP = aminoterminal pro type B natriuretic peptide.

use of NT-proBNP and BNP measurement in the emergency department has been well established [5,6]. In contrast, the use of neurohormones in the outpatient diagnosis of HF has been less well studied. In a study by Groenning et al., NTproBNP measurement in the outpatient setting identified patients with symptoms of $\mathrm{HF}$ and a low left ventricular ejection fraction (LVEF) of less than $40 \%$ with a positive predictive value of 0.11 and a negative predictive value of 1.00 [4]. In a study by Wright et al. that recruited patients presenting to their general practitioner with dyspnea and/or edema, knowledge of the NT-proBNP level improved the diagnostic accuracy of heart failure from $8 \%$ (control group) to $21 \%$ (NT-proBNP group) $(\mathrm{p}=0.002)$ [3]. Finally, Zaphiriou et al. performed a study on 306 patients referred to a heart failure clinic by their general practitioners with suspected heart failure. They found that NT-proBNP and BNP were good rule-out tests with high negative predictive values ( 0.97 and 0.87 , respectively) but low positive predictive values (0.44 and 0.59 , respectively) [2].

Recent research suggests that serial NT-proBNP measurements may have a role in prognostic evaluation [7-12] and therapy optimization [13-16]. In prognostic evaluation, NT-proBNP/BNP has been found to be an independent predictor of sudden death [7], HF hospital admissions [4], and depressed left ventricular function [7]. In a recent systematic review of 19 studies using BNP to estimate the relative risk of death or cardiovascular events in HF patients, BNP was found to be a strong prognostic indicator [18]. Each 100 $\mathrm{pg} / \mathrm{mL}$ increase in BNP was associated with a $35 \%$ increase in the relative risk of death. Further, Maisel et al. in the Rapid Emergency Department Heart Failure Outpatient Trial (REDHOT) study found that BNP is a potent predictor of the 90-day combined event rate of congestive HF visits or admissions and mortality in patients admitted from the emergency department with acute HF [19]. In the Valsartan Heart Failure Trial, HF patients with an initial BNP level greater than $97 \mathrm{pg} / \mathrm{mL}$ were 2.1 times more likely to die at 12 months [20]. As well, in the Australia/New Zealand Heart Failure Study, BNP was superior to LVEF in predicting mortality and HF in patients with ischemic LV dysfunction [9]. Above-median BNP levels conferred a 3-fold-increased risk of mortality. In pre-heart transplant assessment [10], an arbitrary cutoff point of $>2000 \mathrm{pg} / \mathrm{mL}$ for patients with dilated cardiomyopathy and $>1000 \mathrm{pg} / \mathrm{mL}$ for patients with LV dysfunction secondary to coronary artery disease included $90 \%$ of all patients who were clinically considered for cardiac transplantation. As well, BNP appears to be useful in predicting the progression of $\mathrm{HF}$ after cardiac resynchronization therapy (CRT) [21]. Specifically, patients with high BNP values after 1 month of CRT have worse prognosis during follow-up.

Finally, in therapy optimization, Troughton et al. demonstrated that titration of therapy guided by NT-proBNP levels in symptomatic HF patients decreased hospital re-admission rates and cardiac mortality at 9.5 months follow-up, as compared to conventional follow up [14]. BNP may guide the introduction of medications in HF. Richards et al. demonstrated that carvedilol reduced mortality rates in HF to a greater extent in patients with higher pretreatment BNP lev- 
els (9). More recently, in the Systolic Heart Failure Treatment Supported By BNP (STARS-BNP) Multicenter Randomized Trial patients randomized to the BNP guided treatment group suffered fewer events (death or hospitalizations) for HF than in the control group ( $\mathrm{p}<0.001)$ [16].

The above studies suggest that knowledge of an initial NT-proBNP and perhaps repeat NT-proBNP levels may guide diagnosis, prognosis and therapy management. The most recent HF guidelines, specifically, the 2007 Canadian Cardiovascular Society Consensus Conference Recommendations on Heart Failure, support the initial measurement of NT-proBNP/BNP for diagnosis (class I recommendation) and prognostic stratification (class IIa recommendation). The guidelines do not however support sequential measurements of BNP/NT-proBNP levels in the outpatient setting (class IIb recommendation) [17]. To date, no study has demonstrated that routine measurement of NT-proBNP levels in the outpatient setting is cost-effective. In our study, HF specialists were relatively accurate at estimating NT-proBNP levels when a prior NT-proBNP measurement was available, irrespective of the time interval between measurements. In contrast, they were less accurate at predicting NT-proBNP levels in new patients with no prior NT-proBNP level and in patients with patients with a prior NT-proBNP level $\geq 4999$ $\mathrm{pg} / \mathrm{mL}$.

\section{Predictors of NT-proBNP Estimation Accuracy}

Recent studies suggest that NT-proBNP levels are altered in overweight individuals. Specifically, NT-proBNP levels are significantly lower in overweight and obese individuals with HF compared with lean patients [22,23]. Age and gender alter NT-proBNP levels. Redfield et al. found in a recent study that BNP increased significantly with age and was higher in women than men [24]. Renal dysfunction also alters NT-proBNP levels in that eGFR and BNP are inversely related [25].

We hypothesized that age $>65$ years, female gender, elevated BMI $\left(>25 \mathrm{~kg} / \mathrm{m}^{2}\right.$ ), renal dysfunction (eGFR $<60$ $\mathrm{mL} / \mathrm{min} . / 1.73 \mathrm{~m}^{2}$ ), NYHA class I or II, and absence of a prior NT-proBNP level would be predictive of NT-proBNP estimation accuracy. Surprisingly, we found that a prior NTproBNP measurement was the only factor significantly associated with the accuracy of physician estimation. Age $>65$ years, female gender, elevated BMI $\left(>25 \mathrm{~kg} / \mathrm{m}^{2}\right)$, renal dysfunction (eGFR $<60 \mathrm{~mL} / \mathrm{min} . / 1.73 \mathrm{~m}^{2}$ ), NYHA class I or II were not associated with accurate NT-proBNP estimations. In our study, physicians had access to all patient records in the heart failure clinic, including GFR, BMI, etc. We believe that HF specialists purposefully altered their NTproBNP estimation as a result of this knowledge enabling them to guess correctly.

Interestingly, in patients with a prior NT-proBNP level, accuracy reduced greatly as the prior NT-proBNP levels increased. Compared to patients with a prior NT-proBNP level $<125 \mathrm{pg} / \mathrm{mL}$, physicians were $95 \%$ less likely to get a correct estimation, in patients with the highest prior NT-proBNP level $(\geq 4999 \mathrm{pg} / \mathrm{mL})$. Karabulut et al. found that while NTproBNP levels increase significantly with each increasing class of disease, there is a wider range of NT-proBNP levels in NYHA classes III and IV [26]. This may account for our relative inaccuracy at estimating NT-proBNP levels in higher NYHA classes. In these patients, it may be useful to measure NT-proBNP levels on a more routine basis.

\section{CONCLUSIONS}

In conclusion, we found that heart failure specialists are fairly accurate at predicting NT-proBNP levels in heart failure patients only if a prior NT-proBNP measurement is available and the prior level was $<4999 \mathrm{pg} / \mathrm{mL}$. In these patients, likely, initial but not routine measurements of NTproBNP are useful in outpatient heart failure clinics.

\section{ABBREVIATIONS}

Congestive heart failure

Aminoterminal pro type B natriuretic peptide

\section{ACKNOWLEDGEMENTS:}

We would like to acknowledge Drs. Lynn Straatman, Bradley Munt, and Robert Moss, heart failure specialists, who were involved in estimating NT-proBNP levels and have given their permission to be mentioned in this manuscript.

\section{REFERENCES}

[1] Baggish AL, Cameron R, Anwaruddin S, et al. A clinical and biochemical critical pathway for the evaluation of patients with suspected acute congestive heart failure: the proBNP investigation of dyspnea in the emergency department (PRIDE) algorithm. Crit Pathol Cardiol 2004; 3: 171-176.

[2] Zaphiriou A, Robb S, Murray-Thomas T, et al. The diagnostic accuracy of plasma BNP and NT-proBNP in patients referred from primary care with suspected heart failure: Results of the UK natriuretic peptide study. Eur J Heart Fail 2005; 7: 537-541.

[3] Wright SP, Doughty RN, Pearl A, et al. Plasma amino-terminal pro-brain natriuretic peptide and accuracy of heart-failure diagnosis in primary care: a randomized, controlled trial. J Am Coll Cardiol 2003; 42: 1793-800.

[4] Groenning BA, Raymond I, Hildebrandt PR, et al. Diagnostic and prognostic evaluation of left ventricular systolic heart failure by plasma N-terminal pro-brain natriuretic peptide concentrations in a large sample of the general population. Heart 2004; 90: 297-303.

[5] Januzzi JL Jr, Camargo CA, Anwaruddin S, et al. The n-terminal pro-BNP investigation of dyspnea in the emergency department (PRIDE) study. Am J Cardiol 2005; 95: 948-54.

[6] Maisel AS Krishnaswamy P, Nowak RM, et al. Rapid measurement of B-type natriuretic peptide in the emergency diagnosis of heart failure. N Engl J Med 2002; 347: 161-7.

[7] Berger R, Hulsmann M, Strecher K, et al. B-type natriuretic peptide predicts sudden death in patients with chronic heart failure. Circulation 2002; 105: 2392-2397.

[8] Hulsmann M, Berger R, Sturm B, et al. Prediction of outcome by neurohumoral activation, the six-minute walk test and the Minnesota Living with Heart Failure Questionnaire in an outpatient cohort with congestive heart failure. Eur Heart J 2002; 23: 886-891.

[9] Richards AM, Doughty R, Nicholls MG, et al. Neurohumoral prediction of benefit from carvedilol in ischemic left ventricular dysfunction. Circulation 1999; 99: 786-792.

[10] Rothenburger M, Wichter T, Schmid C, et al. Aminoterminal pro type B natriuretic peptide as a predictive and prognostic marker in patients with chronic heart failure. J Heart Lung Transplant 2004; 23: 1189-1197.

[11] Fisher C, Berry C, Blue L, et al. N-terminal pro B type natriuretic peptide, but not the new putative cardiac hormone relaxin, predicts prognosis in patients with chronic heart failure. Heart 2003; 89: 879-81.

[12] Maisel A, Hollander JE, Guss D, et al. Primary results of the rapid emergency department heart failure outpatient trial (REDHOT). A multicenter study of b-type natriuretic peptide levels, emergency 
department decision making, and outcomes in patients presenting with shortness of breath. J Am Coll Cardiol 2004; 44: 1328-33.

[13] Richards AM, Doughty R, Nicholls MG, et al. Plasma n-terminal pro-brain natriuretic peptide and adrenomedullin: prognostic utility and prediction of benefit from carvedilol in chronic ischemic left ventricular dysfunction. Australia-New Zealand Heart Failure Group. J Am Coll Cardiol 2001; 37: 1781-7.

[14] Troughton RW, Frampton CM, Yandle TG, et al. Treatment of heart failure guided by plasma amino terminal brain natriuretic peptide concentrations. Lancet 2000; 355: 1126-1130.

[15] Morimoto T, Hayashino Y, Shimbo T, et al. Is B-type natriuretic peptide-guided heart failure management cost-effective? Int J Cardiol 2004; 96: 177-181.

[16] Jourdain P, Funck K, Gueffet P, et al. Benefit of BNP plasma levels for optimizing therapy: the systolic heart failure treatment supported by BNP multicenter randomized trial (STARS-BNP). J Am Coll Cardiol 2005 ;45: 3A. (Abst)

[17] Arnold JM, Howlett JG, Dorian P, et al. Canadian cardiovascular society consensus conference recommendations on heart failure update 2007: prevention, management during intercurrent illness, or acute decompensation, and use of biomarkers. Can J Cardiol 2007; 23: 21-45.

[18] Doust JA, Pietrzak E, Dobson A, et al. How well does B-type natriuretic peptide predict death and cardiac events in patients with heart failure: systematic review. BMJ 2005; 330: 625-34.

[19] Maisel A, Hollander JE, Guss D, et al. Primary results of the rapid emergency department heart failure outpatient trial (REDHOT): a multicenter study of B-type natriuretic peptide levels, emergency department decision making, and outcomes in patients presenting with shortness of breath. J Am Coll Cardiol 2004; 44: 1328-33.

[20] Anand IS, Fisher LD, Chiang Y, et al. Changes in Brain Natriuretic Peptide and Norepinephrine Over Time and Mortality and Morbidity in the Valsartan Heart Failure Trial (Val-HeFT). Circulation 2003; 107: 1278-1283.

[21] Pitzalis MV, Iacoviello M, Di Serio F, et al. Prognostic value of brain natriuretic peptide in the management of patients receiving cardiac resynchronization therapy. Eur J Heart Fail 2006; 8: 509514.

[22] Horwich T, Hamilton MA, Foarow GC, et al. B-Type natriuretic peptide levels in obese patients with advanced heart failure. $\mathrm{J}$ am coll cardiol 2006; 47: 85-90.

[23] Daniels LB, Clopton P, Bhalla V, et al. How obesity affects the cut-points for B-type natriuretic peptide in the diagnosis of acute heart failure: results from the breathing not properly multinational study. am heart J 2006; 151: 999-1005.

[24] Redfield MM, Rodeheffer RJ, Jacobsen SJ, et al. Plasma brain natriuretic peptide concentration: impact of age and gender. $\mathrm{J}$ am coll cardiol 2002; 40: 976 - 82 .

[25] Vickery S, Price CP, Rohn RI. B-type natriuretic peptide and amino-terminal proBNP in patients with CKD: relationship to renal function and LVH. Am J Kidney Dis 2005; 46: 610-20.

[26] Karabulut A, Kaplan A, Aslan C, et al. The association between NT-proBNP levels, functional capacity and stage in patients with heart failure. Acta Cardiol 2005; 60: 631-8.

(c) Sedlak et al.; Licensee Bentham Open.

This is an open access article distributed under the terms of the Creative Commons Attribution License (http://creativecommons.org/licenses/by/2.5/), which permits unrestrictive use, distribution, and reproduction in any medium, provided the original work is properly cited. 